\title{
A Quantitative Assessment of the Morphofunctional Activity of the Population of Mast Cells Exposed to Biotechnological Strains of Microorganisms
}

\author{
Natalia I. Sheina, $\mathrm{PhD}, \mathrm{ScD}^{1}$; Elena V. Budanova, $\mathrm{PhD}^{2 *}$; \\ Lyubov I. Mjalina, $\mathrm{PhD}^{1}$; Yury P. Pivovarov, $\mathrm{PhD}, \mathrm{ScD}^{1}$; Viktor V. Korolik, $\mathrm{PhD}, \mathrm{ScD}^{1}$; \\ Lyubov P. Sazonova, $\mathrm{PhD}^{1}$; Valentina V. Kolesnikova, $\mathrm{PhD}^{1}$

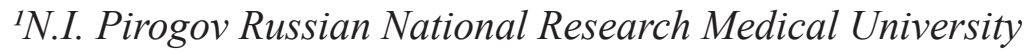 \\ 2I.M. Sechenov First Moscow State Medical University (Sechenov University) \\ Moscow, the Russian Federation
}

\begin{abstract}
In order to assess the sensitizing properties of bacteria, micromycetes and actinomycetes, the morphofunctional activity of the population of mast cells was tested in rats exposed to biotechnological microorganisms. The result showed the high informative value of the test of peritoneal must cell degranulation. Both the result and the intensity of the response of mast cells to the exposure to the tested strains depend on the taxonomy of microorganisms, their concentration and the mode of inoculation. The test of peritoneal must cell degranulation can be recommended for assessing the biological safety of industrial microorganisms. (International Journal of Biomedicine. 2018;8(2):150-154.)
\end{abstract}

Key Words: biotechnology $\bullet$ biotechnological microorganisms $\bullet$ industrial microorganisms $\bullet$ mast cells $\bullet$ biosafety standards

\begin{abstract}
Abbreviations
DI, degranulation index; MC, mast cell; MCD, MC degranulation; PMC, peritoneal MC; SI, saturation index.
\end{abstract}

\section{Introduction}

Biotechnological strains of microorganisms producing the full range of biologically active substances are nowadays widely used in agriculture, medicine, food manufacturing and other areas of our life. However, there is a concern that microbial strains used in biotechnology are potentially hazardous for human health and can increase the risk of allergic reactions and other health issues associated with the use of the recombinant microbial strains or products they can release. In our survey, we tried to evaluate potential sensitizing effects caused by these microbes.

*Corresponding author: Assoc. Prof. Elena V. Budanova, PhD, Department of Microbiology, Virology and Immunology, I.M. Sechenov First Moscow State Medical University (Sechenov University), Moscow, Russia.E-mail: e.v.budanova@mail.ru
Numerous investigations in Russia testify to the extensive influence of biotechnological strains on human health, represented by allergic diseases of the respiratory organs and skin, and by other immune disorders common to people working in the microbiological industry and living in residential areas, even to those people who simply use some products of biotechnology in their everyday lives. ${ }^{(1)}$ That is why controlling and determining the safety of biotechnological strains of microorganisms, and assessing the potential risk of the emission of 'strain producers' and their effect on the population, can help prevent the technogenic pollution of residential areas. This is evidently an extremely important issue.

Methodological approaches for assessing a manifestation of the sensitizing properties of microbial strains used in biotechnology vary greatly. Some of these methods are used in chemical allergology for testing drugs, and the others are 
applied in medical microbiology. However, not all of them are sensitive enough, informative, and suitable for assessing the biosafety of microorganisms used in biotechnology.

As we showed earlier, most biotechnological strains of micromycetes, actinomycetes and typical bacteria are nonpathogenic. Nevertheless, micromycetes and actinomycetes are able to cause the development of type I hypersensitivity (immediate type hypersensitivity), and bacterial biotechnological strains could induce type IV hypersensitivity (delayed type hypersensitivity) in many reported cases.

The biotechnological microbes themselves and the low molecular weight proteins they produce seem to be the main sources of their allergy-inducing activity..$^{(2,3)}$ To demonstrate these possible adverse effects of microbes on the immune system, the whole range of models and in vitro tests can be used: the Shelley's test, the test of the MC degranulation (MCD) and basophil degranulation. ${ }^{(4,5)}$ The MCD test is one of the easy and diagnostically important methods that can be used to detect the potential ability of industrial microorganisms to induce type I hypersensitivity in experiments.

The main aim of our research was to evaluate morphofunctional characteristics of the population of MCs isolated from peritoneal exudate of rats, using the test of the direct MCD, when biotechnological strains of microorganisms are administered into the animal body in different ways.

\section{Material and Methods}

We tested the following nonpathogenic biotechnological strains that produce the biologically active substances: Alcaligenes denitrificans C-32-the producer of nitrilase; Bacillus licheniformis 60 - the producer of a full range of heatstable amylolytic and proteolytic enzymes; Aspergillus awamori Nakazawa VUD T-2 1000-U that releases glucoamylase; Penicillium funiculosum VKM F3668D, as a producer of a group of carbohydrases; Pseudomonas cariophylli KM 92-102/1 that is able to destroy stirols, which is why it is used as a component of biofilters for air purification in the tobacco industry.

The experiments were carried out on conventional male and female white rats (290-320 g, body weight). Each test and control group included 8 animals. Biotechnological strains of microorganisms mentioned above were given to animals by inhalation or were inoculated into the stomach directly using a gastric catheter. The animals were inoculated with minimal effective doses and effective doses of microbes during one month. The animals in the control group were given sterile saline within the same period of time. After the priming, $5.0 \mathrm{ml}$ of sterile Medium 199 was injected intraperitoneally into animals of all the experimental and control groups. Then $7.0 \mathrm{ml}$ of total volume involved peritoneal fluid and Medium 199 was taken through the incision along the linea alba of the abdomen of each rat; next, $0.05 \mathrm{ml}$ of the PMC suspensions were applied on the glass slides and then stained with a $0.03 \%$ solution of neutral red. Peritoneal fluid was examined because the procedure of enriching white cells suspension by basophilic granulocytes is of great complexity and, secondly, because MCs of peritoneal fluid contain a higher histamine level in comparison with mesenteric MCs.
The Institutional Ethical Committee of Animal Care and Use of Pirogov Russian National Research Medical University approved all procedures involving animals. All the procedures of inoculation of animals for collecting peritoneal fluid were performed under sedation of rats with the mixture of zolithyl and xylazine. After finishing each experiment, the animals were euthanized to prevent unnecessary suffering according to recommendations of the Ethical Committee of Animal Care and Use of Pirogov Russian National Research Medical University using an overdose of sodium pentothal.

In order to identify sensitizing properties of the tested biotechnological strains, $100 \mathrm{MCs}$ observed in a field of view were counted under the microscope at different stages of the secretion process. The evaluation of the results was based on the following criteria: ${ }^{(2,6)}$

-The characterization of a cytogram involved the quantity and the degree of color of the metachromatic granules of MC (\%): 1 - dark granules; 2 - dark granules with a visible cell nucleus; 3 - light color of degranulated MCs, 4 -very light color of degranulated MCs with impaired cytolemma (Figures 1-4)

-The frequency (\%) of the detection of degranulated MCs with a different degree degranulation (low, moderate, or high)

-SI, which is calculated as a ratio of the total number of all granulated forms of MCs to the sum of all degranulated MCs. SI indicates the saturation level of the population of MCs with the biologically active substances.

-DI, which represents a ratio of the number of degranulated cells to all MCs identified. DI shows the level of the release of biologically active substances from MCs.

Thus, the criteria listed above show the functional activity of PMC.

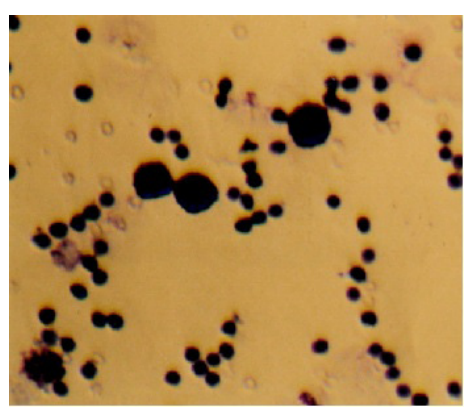

Fig. 1. Very dark MCs depositing metachromatic granules.

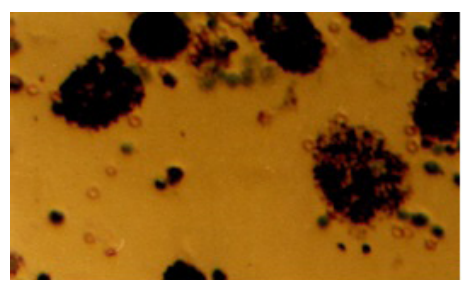

Fig. 2. Very dark MCs with the visible cell nucleus. 


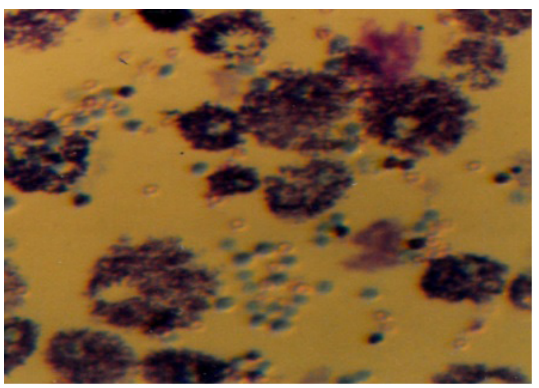

Fig. 3. The light color degranulated MCs.

\section{Results and Discussion}

The results of experiments have shown that there are several distinct stages in the development of the MC response to biotechnological strainsof microorganisms as sensitizing agents. This response depends on the species of microbe and the mode of inoculation, and has a strong «dose-effect» relation. The results are plotted on graphs for individual strains of microorganisms (Figures 5-9).

In the control group of animals, there is an evident balance between dark cells and light colored cells in the peritoneal cytogram, with the dark PMC being prevalent. About $20 \%$ of the cells are degranulated, and most of them show weak to moderate secretion. This fact is set out on the graph as a V-shaped curve with a prominent left side. A similar result was obtained when the $\mathrm{MC}$ population was under the influence of ineffective concentrations/ doses of B.licheniformis 60 (Figure 5).

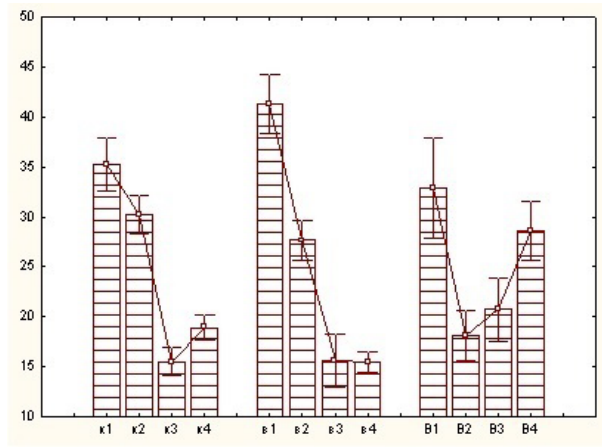

Fig. 5. The stages of the secretion process of the MC population when B. licheniformis 60 is administered through inhalation. The abscissa: $\kappa$ - control; в-concentration of bacteria $5 \times 10^{4} \mathrm{CFU} / \mathrm{m}^{3}$; $B$ - concentration $5 \times 10^{5} \mathrm{CFU} / \mathrm{m}^{3} ; 1,2,3,4$ - stages of secretion of MCs. The Y-axis: concentration of MCs, \%.

The threshold on the sensitizing effect of animals to the microbes inoculated by inhalation was $4 \times 10^{4} \mathrm{CFU} / \mathrm{m}^{3}$ for $A$. denitrificans $10^{4} \mathrm{CFU} / \mathrm{m}^{3}$ for P. funiculosum, $2 \times 10^{4} \mathrm{CFU} / \mathrm{m}^{3}$ for A. awamori, and for $B$. licheniformis $5 \times 10^{5} \mathrm{CFU} / \mathrm{m}^{3}$ of ambient air, respectively. The inoculation via the gastrointestinal tract helped to demonstrate that the sensitizing effect of the microorganisms was limited to $10^{5} \mathrm{CFU} / 1$ for A.denitrificans, $4 \times 10^{5} \mathrm{CFU} / 1$ for $P$. caryophylii, and $2 \times 10^{5} \mathrm{CFU} / 1$ for $B$. licheniformis. When minimum effective concentrations of

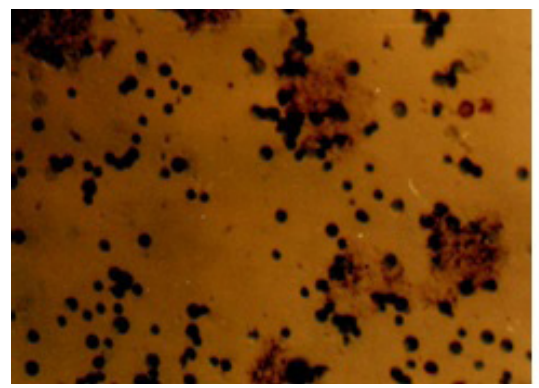

Fig. 4. Severely degranulated MCs, very light in color, with impaired cytolemma.

microorganisms were administered, the population of MCs shifted to the right, towards light colored degranulated cells. The character of the curve showing the functional activity of $\mathrm{MC}$ changes, and the graphs take a V-shape with a prominent and long right «shoulder» (Figures 6 and 7).

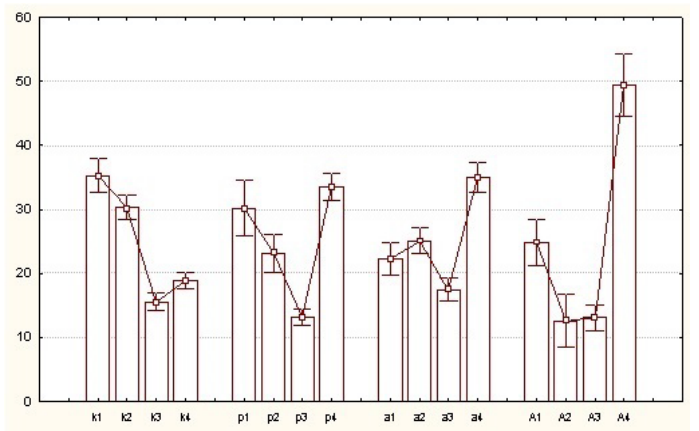

Fig. 6. The stages of the secretion process of the MC population when industrial micromycetes are administered through inhalation. The abscissa: $\kappa$ - control, $p-P$. funiculosum VKM F3668D in concentration of $10^{4} \mathrm{CFU} / \mathrm{m}^{3} ;$ a - A.awamori Nakazawa VUD $T-2$ 1000-U in concentration of $2 \times 10^{4} \mathrm{CFU} / \mathrm{m}^{3} ; A-A$. awamori Nakazawa VUD T-2 1000-U in concentration of $2 \times 10^{5} \mathrm{CFU} / \mathrm{m}^{3}$ of ambient air respectively; 1, 2, 3, 4-stages of secretion of MCs. The $Y$-axis: concentration of MCs, $\%$.

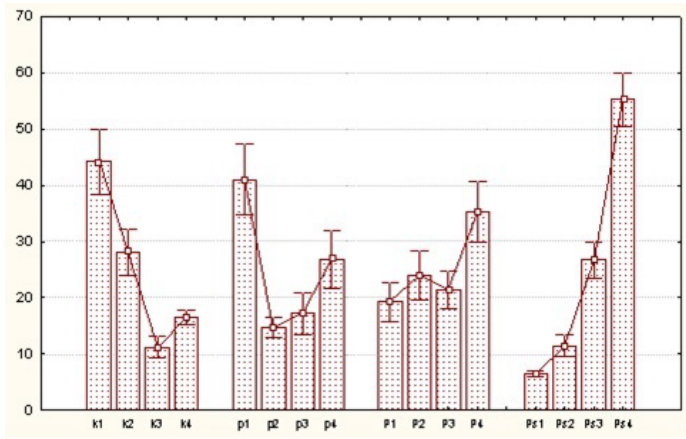

Fig. 7. The stages of the secretion process of the MC population when $P$. caryophylli KM 92-102/1 is administered intragastrically. The abscissa: $\kappa$-control; $p$-concentration of bacteria $4 \times 10^{5}$ CFU/l; $P$ - concentration $10^{6} \mathrm{CFU} / \mathrm{l}$; Ps - concentration $4 \times 10^{7} \mathrm{CFU} / \mathrm{l} ; 1,2,3$, 4 -stages of secretion of MCs. The Y-axis: concentration of MCs, $\%$.

When the amount of a harmful allergy-inducing agent increases (i.e. an administered dose becomes effective), 
significant changes in the cytogram can be observed: The number of dark and oversaturated forms of MC falls off, and the number of empty cells increases. The rate of the curve also changes: The graph takes its V-shape with a high right side.

One should stress that high concentrations of microbes cause pronounced changes in almost all examined indexes, thus, the dose-dependent effect exists.

There is also a tendency for changes to occur in a pool of degranulated MCs when biotechnological microorganisms are administered (Figures 8 and 9). The number of degranulated MCs with a low, moderate and high degree of degranulation is approximately equal in rats of the control group. $\mathrm{MCD}$ progresses if the concentration of the microbes of experimental groups is increased. As the amount of sensitizing agent grows, the number of the strongly degranulated forms increases sharply and both low- and moderate-degree degranulated cells decrease respectively (Figure 9). Thus, the level of the release of biologically active substances from MCs increases in the presence of high concentrations of tested microbes.

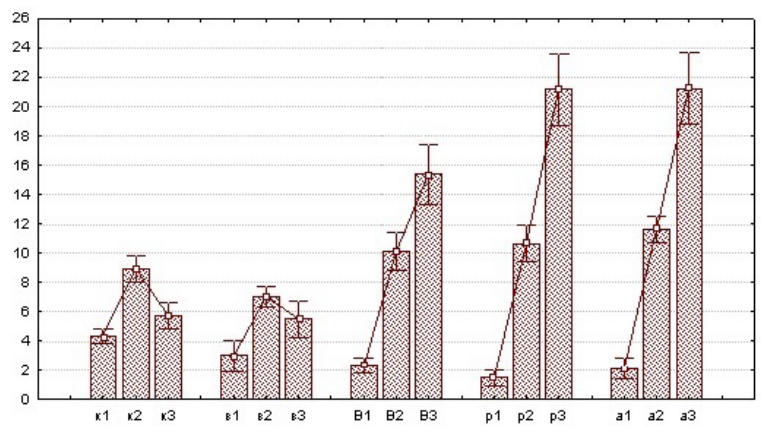

Fig. 8. The main features of the pool of degranulated MCs when biotechnological microorganisms are administered through inhalation. The abscissa: $\kappa$-control; $\beta-B$. licheniformis 60 in concentration of $5 \times 10^{4} \mathrm{CFU} / \mathrm{m}^{3} ; \mathrm{B}-\mathrm{B}$. licheniformis 60 in concentration of $5 \times 10^{5} \mathrm{CFU} /$ $\mathrm{m}^{3} ; \mathrm{p}-$ P. funiculosum VKM F3668D in concentration of $10^{4} \mathrm{CFU} / \mathrm{m}^{3}$; a-A. awamori Nakazawa VUD T-2 1000-U in concentration of $2 \times 10^{4}$ $\mathrm{CFU} / \mathrm{m}^{3} ; 1$ - low degree of degranulation of MCs, 2 - moderate degree of degranulation of MCs, 3 - high degree of degranulation of MCs. The Y-axis: concentration of MCs, $\%$.

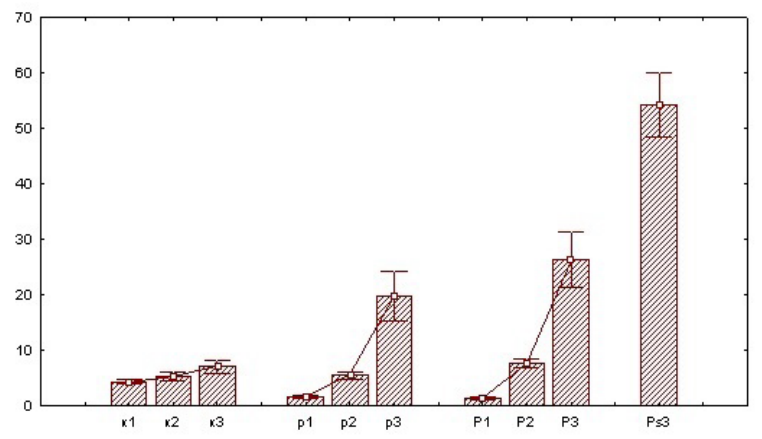

Fig. 9. The main features of the pool of degranulated MCs when $P$. cariophylli KM 92-102/1 is administered intragastrically. The abscissa: $\kappa$-control; $p$-bacteria in concentration of $4 \times 10^{5} C F U / l ; P$ - concentration $10^{6} \mathrm{CFU} / \mathrm{l}$; Ps - concentration $4 \times 10^{7} \mathrm{CFU} / \mathrm{l} ; 1$ - low degree of degranulation of MCs, 2 - moderate degree of degranulation of MCs, 3 - high degree of degranulation of MCs. The Y-axis: concentration of MCs, $\%$.
Table 1 presents SI - an indicator of the functional activity of the population of PMCs, including their ability to synthesize and accumulate histamine. Thus, the SI of intact animals was 2.0. Administration of the threshold concentrations/doses of biotechnological microorganisms tends to lower the histamine concentration in MCs, thus, the index decreases to 1.0. But the effective concentrations/doses of operating microbes cause the release of histamine and other biologically active substances from MCs, and SI drops lower than 1.0.

\section{Table 1.}

The saturation index (SI) of PMCs of rats when biotechnological microorganisms are administered into body through inhalation and intragastrically

\begin{tabular}{|c|c|c|c|c|}
\hline \multirow{2}{*}{ Microorganisms } & \multicolumn{2}{|c|}{ Inhalation } & \multicolumn{2}{|c|}{$\begin{array}{c}\text { Intragastric } \\
\text { administration }\end{array}$} \\
\hline & $\begin{array}{c}\text { Concentration, } \\
\mathrm{CFU} / \mathrm{m}^{3}\end{array}$ & SI & $\begin{array}{l}\text { Dose, } \\
\text { CFU/l }\end{array}$ & SI \\
\hline Control & 0 & $2.3 \pm 0.2$ & 0 & $2.0 \pm 0.1$ \\
\hline $\begin{array}{l}\text { Bacillus } \\
\text { licheniformis } 60\end{array}$ & $\begin{array}{l}5 \times 10^{4} \\
5 \times 10^{5}\end{array}$ & $\begin{array}{l}2.4 \pm 0.4 \\
1.3 \pm 0.4\end{array}$ & $\begin{array}{l}2 \times 10^{5} \\
2 \times 10^{7}\end{array}$ & $\begin{array}{c}1.0 \pm 0.2 * \\
0.5 \pm 0.1 * * *\end{array}$ \\
\hline $\begin{array}{l}\text { Alcaligenes } \\
\text { denitrificans } \\
\text { C-32 }\end{array}$ & $\begin{array}{l}4 \times 10^{4} \\
4 \times 10^{5}\end{array}$ & $\begin{array}{c}1.4 \pm 0.2^{*} \\
0.9 \pm 0.1 * *\end{array}$ & $\begin{array}{l}10^{4} \\
10^{5} \\
10^{6}\end{array}$ & $\begin{array}{c}2.0 \pm 0.3 \\
1.4 \pm 0.3 \\
1.2 \pm 0.1 *\end{array}$ \\
\hline $\begin{array}{l}\text { Pseudomonas } \\
\text { caryophylli } \\
\text { KM 92-102/1 }\end{array}$ & - & - & $\begin{array}{c}4 \times 10^{5} \\
10^{6} \\
4 \times 10^{7}\end{array}$ & $\begin{array}{c}1.6 \pm 0.6 \\
0.9 \pm 0.2 * * \\
0.2 \pm 0.02 * * *\end{array}$ \\
\hline $\begin{array}{l}\text { Penicillium } \\
\text { funiculosum } \\
\text { VKM F3668D }\end{array}$ & $2 \times 10^{5}$ & $1.2 \pm 0.1 * *$ & - & - \\
\hline $\begin{array}{l}\text { Aspergillus } \\
\text { awamori } \\
\text { Nakazawa VUD } \\
\text { T-2 1000-U }\end{array}$ & $\begin{array}{l}10^{4} \\
10^{5}\end{array}$ & $\begin{array}{c}0.9 \pm 0.1^{* *} \\
0.7 \pm 0.1^{* * *}\end{array}$ & $\begin{array}{l}4.4 \times 10^{5} \\
4.4 \times 10^{7}\end{array}$ & $\begin{array}{c}0.9 \pm 0.1^{* *} \\
0.6 \pm 0.1^{* * *}\end{array}$ \\
\hline
\end{tabular}

$* P<0.05, * * P<0.01, * * * P<0.001$

Our experiment has demonstrated that the changes described above depend on the taxonomy of the industrial microorganism and the mode of its inoculation. For example, micromycetes have stronger sensitizing properties than the microorganisms of the other taxonomic groups. In addition, the microorganisms administered intragastrically induced a stronger effect in the targeted MCs than when they were inhaled.

Based on the obtained data, one can claim that the criteria used as indicators for the characterization of MC populations has high informative value. The morphofunctional analysis of PMC includes not only the assessment of secretory activity of individual cells but also the functional activity of whole populations of cells. These criteria can be applied to the quantitative assessment of the allergenic activity of chemicals and microbial strains used in biotechnology. They can be especially useful in the presence of low doses of potential allergens.

As it has been shown previously, the formation of MCs and the dynamics of their functional activity can be a useful sensitive indicator for the identification of even mild allergic processes. ${ }^{(6,7)}$ Such analysis of MC populations, for example, helps in determining the sensitizing effect of natural rubber or latex and some other chemicals, which is observed in people 
but cannot be seen in animals. Indeed, basophils and MCs of body fluids and tissue basophils become very sensitive models that can be used to detect the increased risk in the development of type I hypersensitivity. ${ }^{(8)}$ It is well known that the membranes of MCs and basophils have FceRI receptors to IgE that results in the release of histamine. It seems to be the main mechanism of the initiation and development of type I hypersensitivity. Thus, MCs, basophils, and eosinophils are central effector cells in allergic inflammation, as well as in innate and adaptive immunity. ${ }^{(8,9)}$ One should emphasize that the development of type I hypersensitivity occurs due to the activation of targeted MCs, their degranulation, and the release of biologically active substances not due to the death of the cells.

To summarize, the assessment of morphofunctional activity of MCs and the characterizing of their population could serve as a useful test for revealing a potential risk in the development of allergic reactions caused by biotechnological microorganisms or products they can synthesize. The experimental data of our survey have identified the limit of concentrations of the microbial industrial strains that can induce possible adverse effects: It is above $4 \times 10^{4} \mathrm{CFU} / \mathrm{m}^{3}$ of ambient air through inhalation and over $10^{5} \mathrm{CFU} / 1$ if administered intragastrically. These doses of microbes given continuously within one month can lead to prominent changes in the composition and functional activity of pools of MCs and can dramatically affect tissue homeostasis of animal organisms in experiments.

\section{Conclusions:}

1. The morphofunctional analysis of MC populations must include the assessment of secretory activity of both individual cells and the whole population.

2. SI and DI of PMCs can serve as a diagnostic tool used to detect the possible allergy-inducing activity of chemicals and industrial strains of microbes. This test is very necessary in assessing the safety of biotechnological microorganisms.

3. The severity and the nature of the changes depend on the taxonomic category of the biotechnological microbial strain and the mode of inoculation, and have the prominent dose-dependent effect.
4. Thus, the quantitative assessment of morphofunctional characteristics of the population of PMC using the MCD test can be applied to reveal a specific allergenic effect of biotechnological microorganisms, even in very low concentrations.

\section{Competing interests} interests.

The authors declare that they have no competing

\section{References}

1. Sheina NI, Budanova EV, Pivovarov YuP, Mjalina LI, Sazonova LP. Biosafety assessment of microbial strains used in biotechnology according to their taxonomy. International Journal of Biomedicine. 2017;7(1):51-56.

2. Fedoseyeva VN, Poryadin GV, Kovalchuk LV, Cheredeev AN, Kogan VYu. A guide on immunological and allergological methods used in hygienic researches. Moscow: Promedek; 1993. [In Russian].

3. Fedoseyeva VN, Molotilov BA, Larina ON. Bacterial allergy. Penza, 2004. [In Russian].

4. Protsenko VA, Shpak SI, Dotsenko SM. Tissue basophils and basophilic granulocytes of the blood. Moscow: Meditsina; 1987. [In Russian].

5. Lindner DP, Pobery IA, Rozkin MYa, Yefimov VS. [Morphometric analysis of a population of the mast cells]. Arkh Patol. 1980;62(6):60-64. [Article in Russian].

6. Berezhnova LI, Petrova LP. [Dynamics of the must cell count and functional activity in the skin of guinea pigs as an index of mildly expressed allergic processes]. Vestn Dermatol Venerol. 1982;(12);56-60. [Article in Russian].

7. Duyeva LA, Karamysheva AV. [Reaction of specific degranulation of mesenterial mast cells of rats and guinea pigs as a new test used for the assessment of allergenic activity of chemicals]. Tox Vestn. 2001;4:19-23. [Article in Russian]. 8. Stone KD, Prussin C, Metcalfe DD. IgE, mast cells, basophils, and eosinophils. J Allergy Clin Immunol. 2010;125(2 Suppl 2):S73-80. doi: 10.1016/j.jaci.2009.11.017.

9. Pawankar R, Yamagishi S, Takizawa R. Mast cell-IgE and mast cell-structural interactions in allergic airway disease. Curr Drug Targets Inflamm Allergy. 2003;2(4):303-12. 